\title{
The photomyoclonic reflex: an artefact in the clinical electroretinogram*
}

\author{
MARY A. JOHNSON AND ROBERT W. MASSOF \\ From the Wilmer Ophthalmological Institute, the Johns Hopkins University School of Medicine, \\ Baltimore, Maryland, USA
}

SUMMARY An artefact may appear in the clinical electroretinogram (ERG) that can interfere with the recording and interpretation of the ERG $b$ wave. This artefact, the photomyoclonic reflex (PMR), was studied by covering the eye containing the recording electrode and stimulating the fellow eye. Records obtained by this technique before and after administration of a modified Van Lint lid block demonstrated that most of the PMR is due to a reflex contraction of the orbicularis muscle. The remaining part of the PMR was ascertained by eye movement recordings to be a $1.5^{\circ}$ to $3.5^{\circ}$ downward and medial eye movement. In most persons the PMR occurs with a latency that is fast enough ( $59 \mathrm{~ms} \pm 7 \mathrm{~ms}$ ) to interfere with interpretation of the $b$ wave under most conditions. The PMR can be minimised in some cases by habituation or conditioned suppression. However, these methods generally do not extinguish the PMR but reduce it enough so that it would not readily be rejected as artefact. In such cases the PMR may produce a wave form that mimics a normalamplitude ERG but with delayed implicit time.

The electroretinogram (ERG) is a light-evoked gross electrical potential generated by retinal cells and recorded at the cornea. Clinically the ERG is often employed to gain objective information about the status of the neurosensory retina.

Although much has been learned in recent years about the cellular origins of various ERG components, there still is considerable variability in the ERG wave form that reflects significant contributions to the recorded potential from extraretinal sources. For example, Gur and Zeevi, ${ }^{1}$ in the course of performing frequency analyses on the human ERG, found that the signal is time-locked to the stimulus only during the first 55 to $60 \mathrm{~ms}$ of the response. At that time an additional component, which was not completely time-locked to the stimulus, interfered with the ERG potental. Gur and Zeevi attributed this component to a light-induced eye movement, a photomyoclonic reflex (PMR). They cited unpublished experiments by Bickford $e t$ al. ${ }^{2}$ that were

*Presented at the 1980 Annual Meeting of the Optical Society of America in Chicago. Illinois, and at the 1981 Annual Meeting of the Association for Research in Vision and Ophthalmology in Sarasota, Florida.

Correspondence to Dr R. W. Massof, Wilmer Institute, Rm. B-34, Johns Hopkins Hospital, Baltimore, MD 21205, USA. said to demonstrate a reflex clonus of the eye musculature in response to a bright flash of light.

The interference from a muscular light-reflex artefact in the recording of the ERG was first noted by Karpe. ${ }^{3}$ In his classic monograph on clinical electroretinography Karpe demonstrated that lightinduced reflex 'blinking' can significantly impair ERG recordability and interpretation. For example, using a contact lens electrode and a spring-loaded speculum he attempted to record an ERG from a retinitis pigmentosa patient and obtained a small 'blink artefact,' in the absence of any retinal potentials, which resembled a normal ERG except for its delayed timing.

The existence of reflexive, light-induced blinks is well known to clinical electroretinographers. When the blink artefacts are large, they are readily recognised in ERG records. However, less apparent eye movements that accompany all blinks ${ }^{4}$ may also interfere with ERG recordings. ${ }^{5}$ These eye movements, which can occur when the lid reflex is small or has been aborted, ${ }^{4}$ vary in amplitude and thus could be subtle enough to go clinically unnoticed.

Using the time-locking criterion of Gur and Zeevi ${ }^{1}$ we have been able to identify retrospectively a PMR artefact in nearly all the records from our clinical 

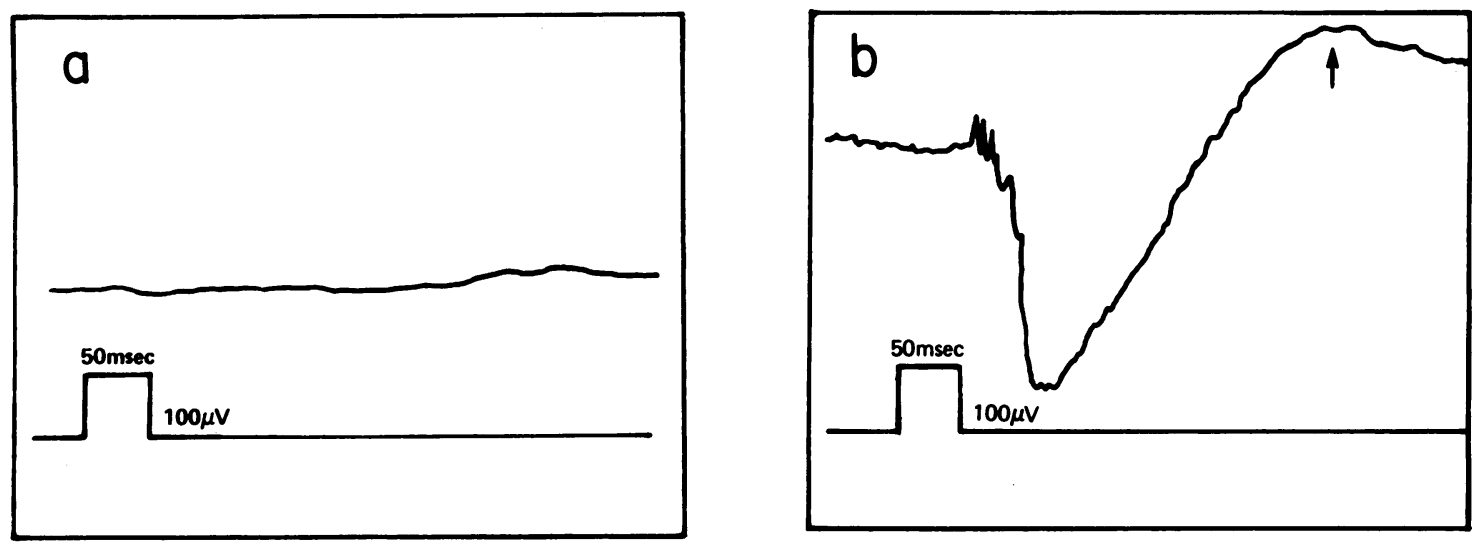

Fig. 1 A photomyoclonic reflex (PMR) was recorded, uncontaminated by retinal potentials, by occluding the eye containing the electrode and stimulating the fellow eye. (a) Control condition; no response was recorded when both eyes were covered. (b) A PMR, recorded from the covered experimental eye. The PMR waveform typically consists of spiking followed by a fast corneal-negative, then slow positive, phase (arrow). This reflex has a latency of $55 \mathrm{~ms}$. In all Figures the $10 \mu \mathrm{s}$ stimulus occurs at the rising edge of the calibration pulse.

ERG laboratory. Furthermore we have been able to recognise this artefact in several published ERG records from other laboratories. Therefore the present study was conducted to characterise the PMR further and to determine its artefactual contributions to the clinical ERG. Some of the studies reported here are replications of experiments that were designed to investigate other problems or in some cases were technically unsatisfactory. Our results indicate that the PMR artefact can be subtle and can significantly interfere with the interpretation of the clinical ERG.

\section{Materials and methods}

ERGs and PMRs to a full-field (Ganzfeld) stimulus were elicited by a $10 \mu$ s white flash from a Grass PS-22 variable-intensity stimulator. The luminance for a maximal intensity $(I=16)$ stimulus was $-0.26 \log \mathrm{cd}$. s. $\mathrm{m}^{-2}$. A Burian-Allen bipolar electrode wetted with methylcellulose was used to record the evoked response. The signals were amplified alternatingcurrent coupled $(0 \cdot 1 \mathrm{~Hz}$ to $300 \mathrm{~Hz})$ amplifiers. The responses were displayed on a storage oscilloscope, and photographed with a Tektronix C5 oscilloscope camera.

Mydriasis was obtained with $1 \%$ tropicamide and $10 \%$ phenylephrine hydrochloride. A $0.5 \%$ solution of proparacaine hydrochloride was used in all cases as the corneal anaesthetic.

Two neutral-density filters of 1 and $2 \log$ units (nominal) attenuation were used in conjunction with different intensity settings on the photostimulator to determine the intensity-response characteristics of the PMR.
Latencies of PMRs were measured retrospectively from the records of 97 patients who had normal amplitude ERGs. Subjects for the different experimental manipulations included the 2 authors, 2 other normal volunteers, and 3 volunteers who have retinitis pigmentosa (RP). These volunteers gave their informed consent to the experiments.

\section{Results}

\section{Properties of the PMR}

Six experiments that characterise properties of the PMR are described in this section. For most experi-

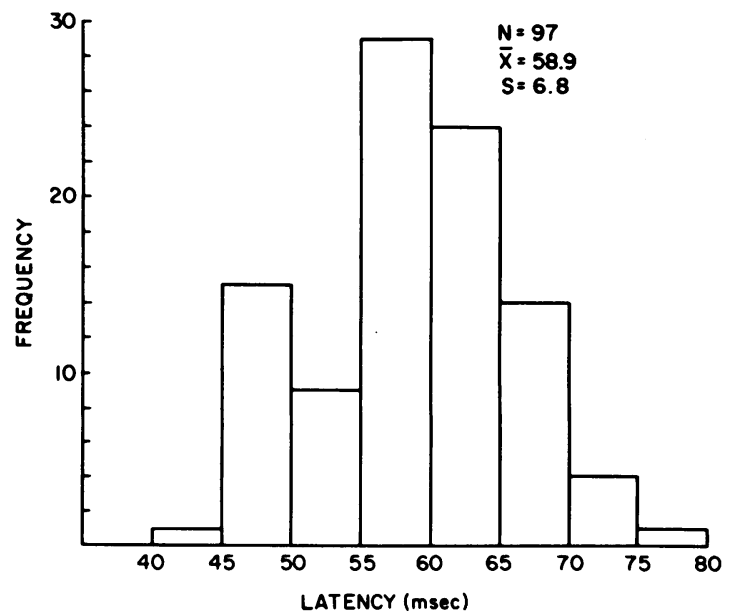

Fig. 2 Frequency distribution of PMR latencies (mean $=59$ $\mathrm{ms}, S D=7 \mathrm{~ms}$ ) measured from $E R G$ records of 97 patients with normal ERGs. 

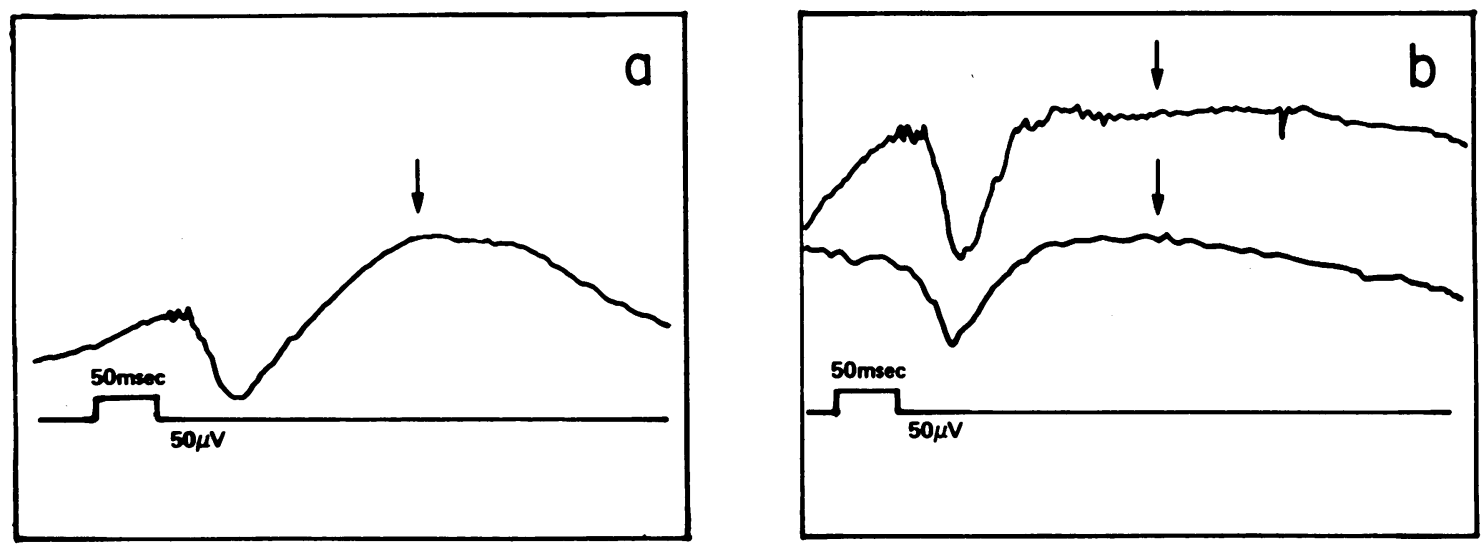

Fig. 3 The pupillary reflex is the source of the late positive wave of the PMR. (a) Arrow points to the late positive phase of a standard-recorded PMR with normal, reactive pupils. (b) After mydriatics were instilled 2 PMRs recorded from the subject lacked the late positive waves. Arrows point to areas of the PMR occurring at same point in time as that in (a).

ments, in order to record the PMR uncontaminated by retinal potentials, the eye with the electrode was occluded and the fellow eye was stimulated.

\section{QUALITATIVE FEATURES OF THE PMR}

This experiment describes the PMR waveform unobstructed by the ERG.

Procedure. After 20 minutes' dark adaptation a Burian-Allen bipolar ERG electrode was inserted. The eye was then occluded by a cone covered with black photographic tape to prevent exposure to light during the study. To verify the opacity of the cone recordings were obtained with both eyes occluded. The subject sat in a shielded room with his head positioned on a chin rest facing an integrating sphere. A red light-emitting diode (LED) served as a fixation point for the unoccluded eye.

Results. Fig. 1a illustrates the results of the control experiment. No response was recorded when both eyes were occluded. Fig. 1b illustrates the response recorded in the occluded eye during stimulation of the fellow eye. The large corneal-negative deflection has a latency of $55 \mathrm{~ms}$ in this subject. The negative deflection is usually, but not always, preceded by spiking activity, and is followed by a slower, positive wave (arrow) that has an average latency of about 160 ms.

The latencies of PMRs measured retrospectively from ERG records of 97 normal patients are presented in Fig. 2. The distribution is bell shaped; the mean is $58.9 \mathrm{~ms}$ and the standard deviation is 6.8 $\mathrm{ms}$. The range of PMR latencies is $44 \cdot 4$ to $77.8 \mathrm{~ms}$.

\section{PUPILLARY CONTRIBUTIONS TO THE PMR}

The PMR wave form becomes altered when a mydriatic is instilled for ERG recording. The next experiment is designed to assess the contribution of the pupillary light reflex to the PMR.

Procedure. The PMR was recorded with undilated pupils by the procedures of the first experiment. The cone and electrode were then removed and a mydriatic was instilled into the experimental eye. The electrode and cone were replaced, the fellow eye was stimulated, and responses of the experimental eye were recorded.

Results. The long-lasting positive component of the artefact (arrow in Fig. 3a) is abolished by mydriasis of the pupil (arrows in Fig. 3b). Therefore we conclude that this late, slow potential is a pupillary response to the light flash. These results and conclusions are identical to those of Pearlman, ${ }^{5}$ based on his studies of the ERG $\mathrm{c}$ wave.

\section{HABITUATION OF THE PMR}

Repeated stimulus presentations diminished the amplitude of the PMR. The next experiment demonstrates that the amplitude reductions are the result of habituation rather than adaptation.

Procedure. Recordings were obtained simultaneously from the occluded and nonoccluded eyes. Stimulus flashes were presented every 3 seconds to the nonoccluded eye. Forty-five seconds after the flashes were terminated 2 additional flashes, $45 \mathrm{~s}$ apart, were presented.

Results. The ERG from the nonoccluded eye remained constant in amplitude, i.e., no changes in adaptation occurred. Fig. 4a illustrates the responses in the covered eye to 4 successive flashes, $3 \mathrm{~s}$ apart. The first response to the stimulus was the largest, the last the smallest. Fig. $4 \mathrm{~b}$ shows the responses to the next 4 flashes of light. Aside from the late pupillary response, the wave form is almost completely 

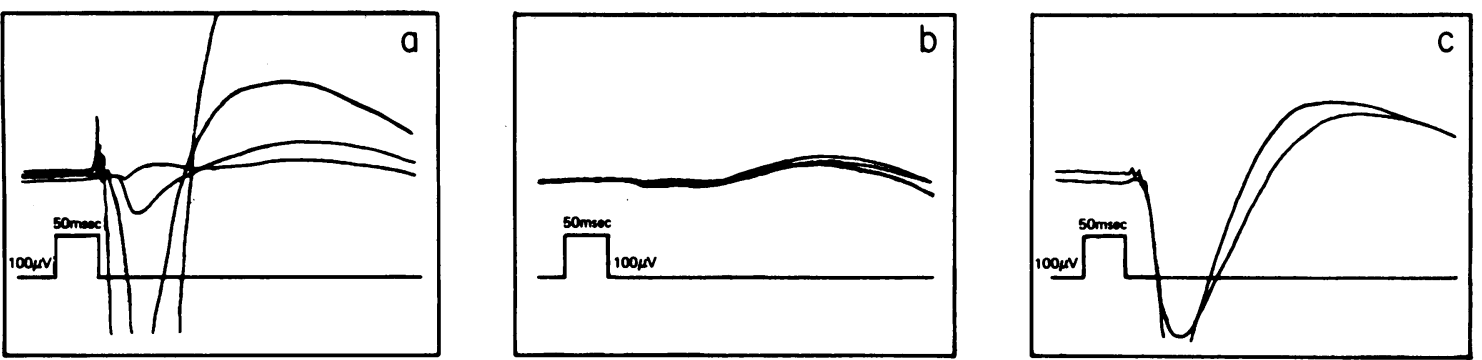

Fig. 4 Habituation of the PMR. (a). Four PMRs, 3 s apart, recorded in standard fashion from one subject. The first response is the largest negative deflection, the second response is the second largest negative deflection, etc. (b) Four responses, 3 s apart, to the next 4 flashes of light show complete habituation of the negative wave of the PMR. The late positive wave is the pupillary reflex. (c) Return of the PMR, recorded 45 s after cessation of habituating stimulus. The 2 responses were recorded 45 s apart.

abolished. Fig. $4 \mathrm{c}$ illustrates 2 responses, $45 \mathrm{~s}$ apart, recorded $45 \mathrm{~s}$ after the records shown in Fig. $4 \mathrm{~b}$. The PMR was again elicited.

The results of this experiment indicate that the PMR becomes habituated with repeated stimulation, despite no apparent changes in retinal adaptation. This habituation presumably is under CNS control.

\section{CONDITIONED SUPPRESSION OF THE PMR}

Recent studies have shown that the blink reflex to tactile stimulation can be suppressed by auditory stimuli. ${ }^{67}$ If the PMR arises by way of a noncortical pathway, as suggested by its short latency, it should not be possible to condition it to a cross-modal stimulus, although response modification by conditioned suppression may be possible.
Procedure. The PMR was recorded from the occluded eye as described previously. A $50 \mathrm{~ms}$ tone was presented, instead of the light, in order to determine whether the PMR could be elicited solely by the tone. The tone then was paired with the light flash, preceding the light by $150 \mathrm{~ms}$, and presented every $3 \mathrm{~s}$ for a period of $15 \mathrm{~min}$. After this training period the tone alone, then the flash alone, and then the tone followed by the flash were delivered to determine the effects of conditioning.

Results. Fig. 5a shows that prior to conditioning the tone alone did not elicit a PMR. Fig. 5b illustrates that the tone alone also failed to elicit a PMR after conditioning. Thus the PMR could not be conditioned to the cross-modal stimulus. Fig. $5 \mathrm{c}$ is the response elicited by light alone after conditioning
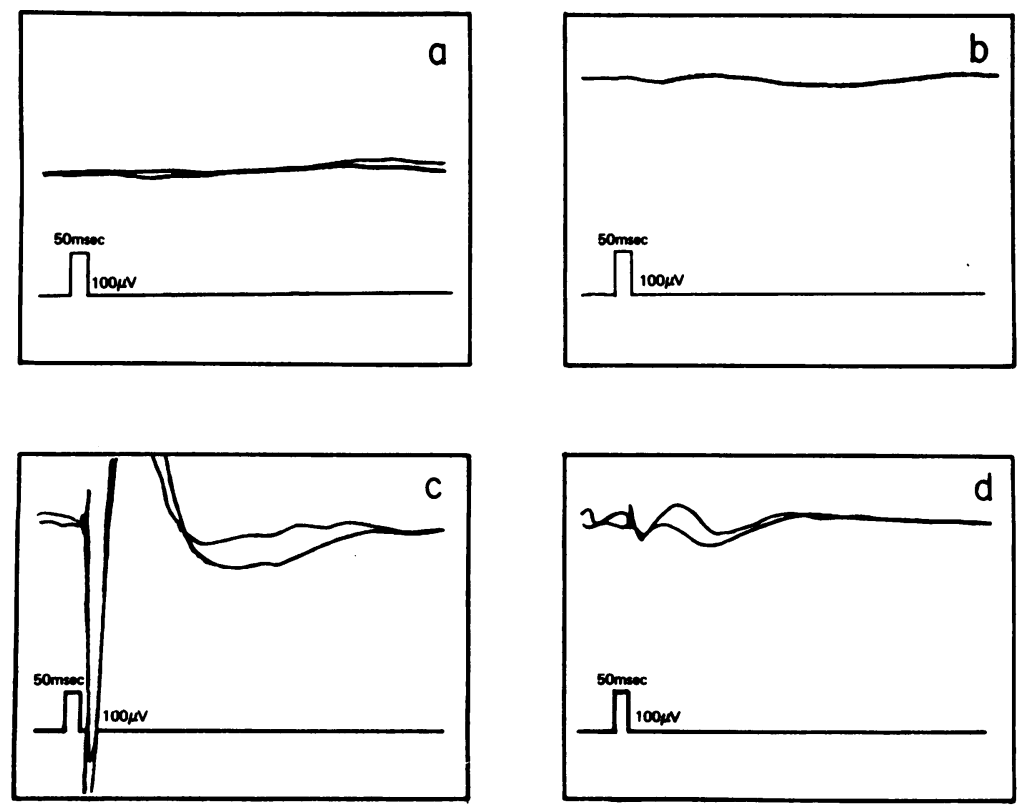

Fig. 5 Conditioned suppression of the PMR by an auditory stimulus. The PMR was recorded in the standard manner. (a) Before conditioning, no reflex was elicited by a $50 \mathrm{~ms}$ tone. (b) The tone was paired with (presented $150 \mathrm{~ms}$ before) a flash of light for about 15 min. After this conditioning no response was elicited by the tone alone. A large-amplitude PMR was elicited by the light only (c), but not when both tone and flash were presented (d). Presence of the tone ahead of the flash suppressed the reflex. 
trials, and Fig. 5d is the response to the light paired with the tone. Figs. 5c and 5d show that, after conditioning, the tone suppressed the PMR, i.e., the PMR is dramatically reduced in amplitude when the light flash is paired with the tone.

These data indicate that the PMR probably has a subcortical origin but can be suppressed by the cortex. In contrast, pupillary reflexes cannot be suppressed in this manner. Furthermore PMR habituation and suppression rule out any putative roles of electrotonic spread of excitation ${ }^{8}$ as the source of the PMR.

\section{CONTRIBUTION OF THE ORBICULARIS TO THE PMR}

Karpe $^{3}$ attributed artefacts such as the PMR to dazzle reflex eyelid movements. Electroretinographers are aware that from time to time 'blinks' occur in ERG records. Bickford ${ }^{2}$ suggested that an eye movement rather than a blink is responsible for variations in the ERG. However, in a study of blink reflexes Miles ${ }^{4}$ noted that eye movements accompany all blinks, actual or aborted.

Rushworth ${ }^{9}$ measured the latencies of over 600 light-induced blink reflexes in 20 patients and found a mean near $60 \mathrm{~ms}$. As we found for the PMR, he observed that the blink could be elicited in the contralateral eye, that it could show habituation, and that it did not become conditioned to an auditory stimulus. The next experiment was designed to determine the contribution of a blink to the PMR.

Procedure. A modified Van Lint lid block was performed on one of the authors with $10 \mathrm{ml}$ of a 50:50 mixture of $2 \%$ lignocaine and $0.75 \%$ bupivacaine, with added hyaluronic acid. The subcutaneous tissues were infiltrated with the anaesthetic solution surrounding the orbital rim and extending back $4 \mathrm{~cm}$ toward the tragus of the ear. This lid block was clinically complete in paralysing the orbicularis muscle within $15 \mathrm{~min}$, resulting in the subject's inability to close the eye, and lasting for about 3 hours.

Prior to the lid block ERG electrodes were placed in both the occluded and nonoccluded eyes, and the ERG and PMR were simultaneously recorded. this procedure was repeated after the orbicularis of the occluded eye was completely paralysed by the lid block.

Results. Fig. 6a shows that the PMR was present in both eyes prior to the lid block. The top trace is the PMR superimposed on the ERG from the unoccluded eye. The bottom trace is the PMR from the occluded, experimental eye. Note that the PMR is consensual.

Fig. 6b illustrates the PMR after the orbicularis muscle of the occluded eye is paralysed. As before, the PMR was elicited in the nonoccluded eye (top trace). As seen in the bottom trace, paralysis of the orbicularis eliminates the spiking and the initial negative potential of the PMR. However, a small corneal-positive potential remains.

These data indicate that the major portion of the initial corneal-negative wave form of the PMR can be attributed to contraction of the orbicularis.
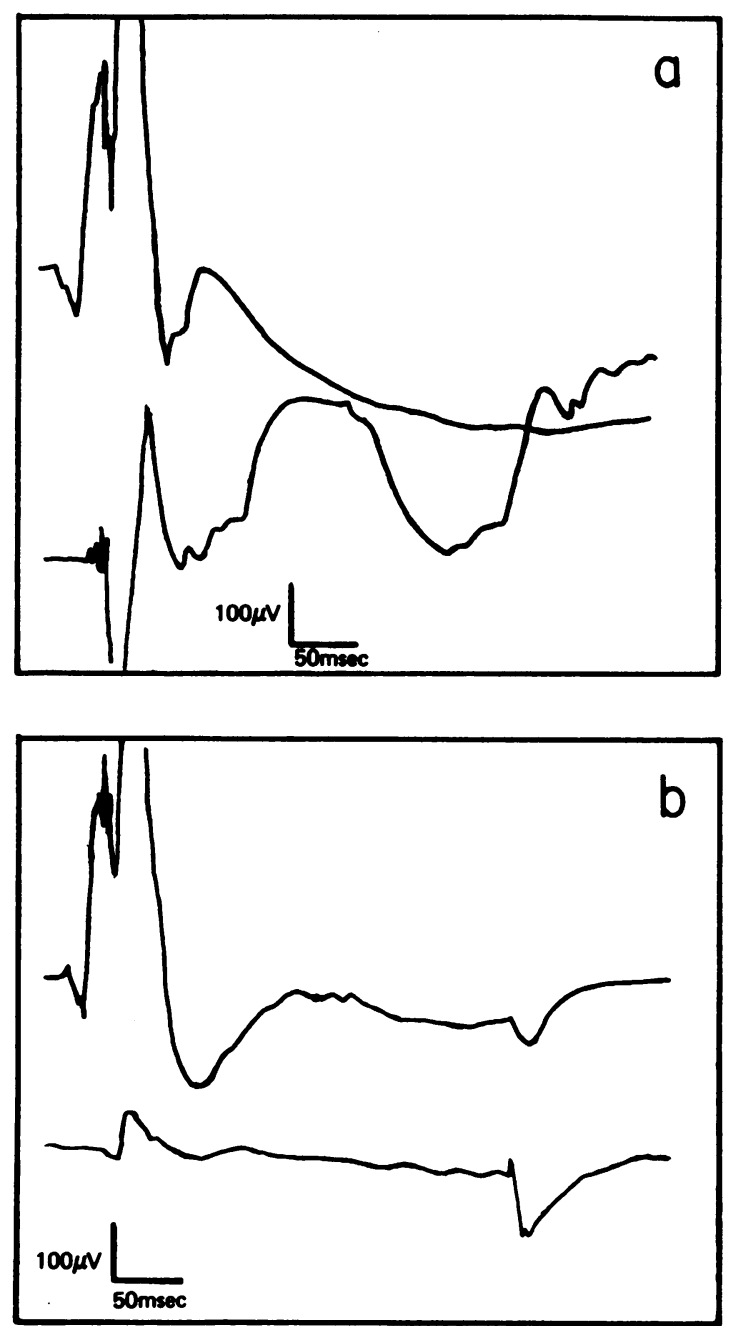

Fig. 6 Role of orbicularis muscle contraction in the PMR. Electrodes were placed on both eyes, and one eye was occluded from the light. (a) The top trace shows the ERG and $P M R$ recorded from one eye, and the bottom trace shows just the PMR recorded from the occluded fellow eye. (b) $A$ modified unilateral Van Lint lid block was then administered to the covered eye. Twenty minutes later the light-elicited responses were again recorded from both eyes under the same conditions. Most of the PMR was eliminated by the lid block (lower trace), indicating substantial contributions from the orbicularis muscle. 


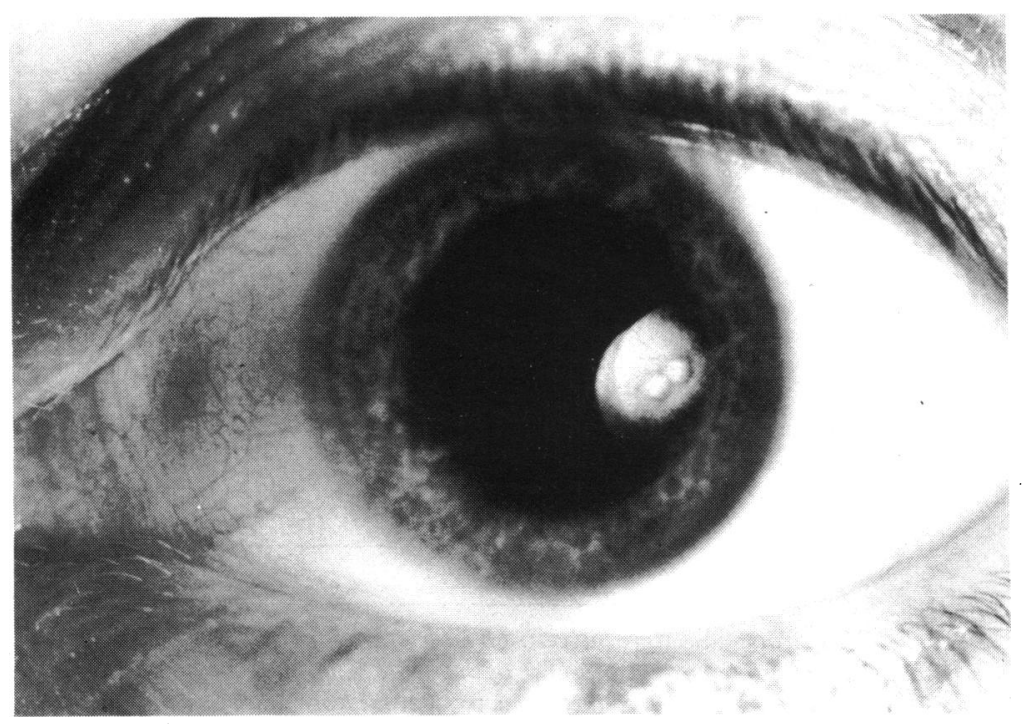

Fig. 7 Double-exposure photograph showing small eye and eyelid movements. Photograph was taken in a dark room with the camera shutter held open. The light source was a strobe, triggered to double flash with an interflash interval of $120 \mathrm{~ms}$. Note the 2 displaced corneal reflections from the strobe and the displaced images of the scleral vasculature and eyelashes.

Furthermore, because the initial spiking was eliminated, we conclude that it too may be attributed to the orbicularis. This latter conclusion is in agreement with Kennard and Glaser, ${ }^{10}$ who attributed spiking in blink reflex records to nervous innervation of the orbicularis muscle.

\section{THE ROLE OF EYE MOVEMENTS IN THE PMR}

The residual response in Fig. $6 \mathrm{~b}$ may be due to a small eye movement. This hypothesis is consistent with the finding by Miles ${ }^{4}$ that all blinks, whether actual or aborted, are accompanied by eye movements. The following experiments examined this possibility.

Procedure. We recorded reflex movement of the eye by photographing the eye before and during the PMR. In a dark room a camera was focused on the subject's eye, and the shutter was opened. A double strobe flash was then delivered to the subject. The interval between flashes was $120 \mathrm{~ms}$, which is roughly the implicit time of the PMR.

An accurate determination of the amplitude and direction of this eye movement was established by the following procedure. A Burian-Allen bipolar ERG electrode was placed on one eye to record the presence of the PMR. The other eye contained a scleral eye coil which, when the subject was placed in a magnetic field, could record eye movements with a resolution of less than 30 minutes of arc (for methods, see Robinson ${ }^{11}$ and Collewijn et al. ${ }^{12}$ ). A strobe flash was used as the stimulus.

Results. The double-exposed photographs showed small movements of the eyelid and eye in the 3 subjects tested. Fig. 7 is a typical photograph from one subject. Note the 2 displaced corneal reflections from the strobe, and also displaced images of the scleral vasculature and the eyelashes. This observation supports the hypothesis that the remnant of reflex after the lid block can be attributed to eye movement.

Data from the scleral coil eye movement recordings are shown in Fig. 8. Figs. 8a and $b$ are records obtained during the same light flash. Fig. 8a is the record from the ERG electrode, which shows that a PMR having a latency of $55 \mathrm{~ms}$ (arrow) occurred in response to the light flash. Record $8 \mathrm{~b}$ from the eye coil shows an eye movement with a latency of $65 \mathrm{~ms}$, occurring downwards and medially. Eye movements from the 2 subjects tested had amplitudes ranging from $1.5^{\circ}$ to $3 \cdot 5^{\circ}$.

These data show that eye movements occur in conjunction with reflex blinks during a PMR. The eye movements have slightly longer latencies and apparently produce smaller potentials than eyelid movements.

\section{Clinical ERG Interpretations and the PMR}

Important considerations for assessing the impact of the PMR on ERG interpretation must include: (1) the timing of the PMR in relation to the various components of the ERG, and (2) the shape of the PMR wave form and how it may distort or mimic the ERG wave form. These aspects of the PMR are explored in the next set of experiments. Also, a brief study comparing the propensity of several popular ERG electrode types to record the PMR is described.

7. INTENSITY-RESPONSE CHARACTERISTICS OF THE PMR

We obtained an intensity-response function for the 


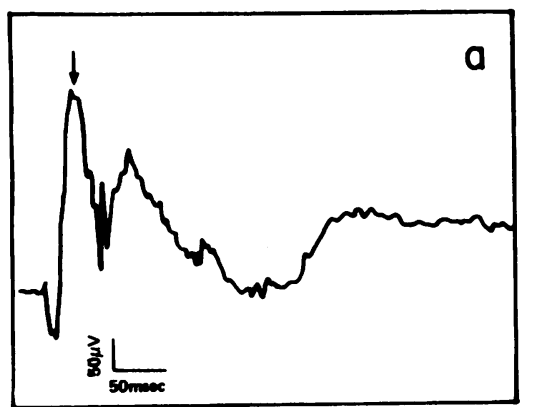

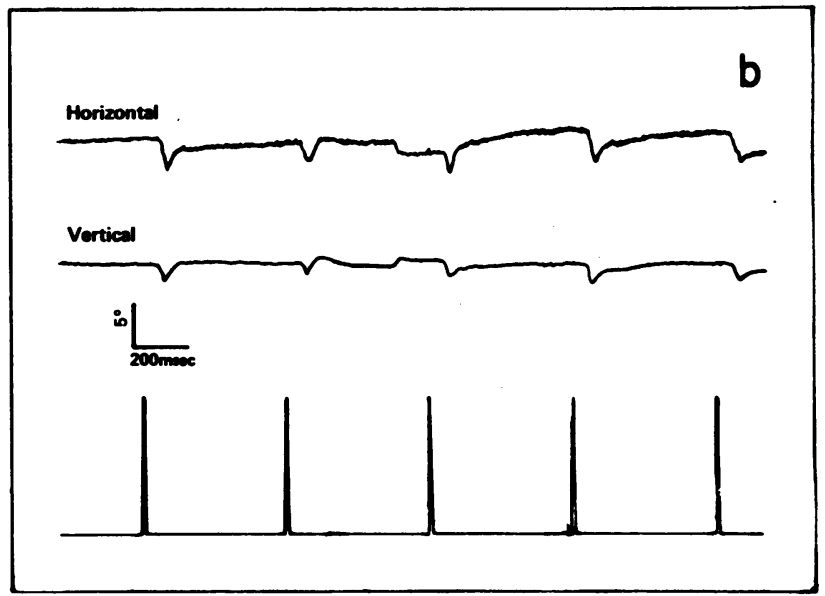

Fig. 8 Extent of eye movements in a PMR elicited by a strobe flash were measured by a scleral eye coil. ${ }^{12}$ (a) Record from a standard ERG electrode which was placed on the fellow eye to record the presence of the PMR. The arrow points to PMR onset. (b) Eye movement recordings. Calibrations were obtained by saccadic pursuit of stepwise horizontal or vertical displacements of a fixation spot. Horizontal and vertical components of the light-elicited reflex eye movement were recorded on 2 separate channels of a pen writer. The horizontal component (up=rightward movement) of the eye movement measured in the subject's right eye has a latency of about $65 \mathrm{~ms}$ and an average amplitude of about $3^{\circ}$ in this subject. The vertical component (up = upward movement) occurs simultaneously with the horizontal component and has an average amplitude of about $2^{\circ}$ in this subject. Consequently the direction of the eye movement was toward $7 o^{\prime}$ clock (downwards and medial) with an amplitude of about $3 \cdot 5^{\circ}$.

PMR in order to determine how the latency and amplitude related to stimulus strength.

Procedure. The PMR was recorded from the occluded eye by the procedures described above. Stimuli of increasing intensity, covering a range of 3 log units, were presented to the unoccluded eye. Results were compared with ERG records obtained at an earlier date from the same subject by means of the same stimuli and order of stimulus presentation.

Results. We found that the amplitude of the PMR does not depend on stimulus strength in any predictable fashion; amplitude depends mainly on the state of habituation. However, there is a PMR threshold, which varied between observers. Latency on the other hand decreased slightly (from 67 to 56 $\mathrm{ms}$ ) over a 3 log-unit increase of intensity (Fig. 9). To determine the effect of PMR latency change (or lack of it) on ERG interpretation, the implicit times (time from stimulus onset to peak of the wavelet) of ERG $b$ waves, obtained under the same stimulus conditions as the PMR, were plotted along with the PMR data. (The $b$ wave implicit time was selected for comparison because the appearance of the $b$ wave in patients usually forms the basis for ERG evaluation.) As seen in Fig. 9, the graphs of the 2 functions cross at $57 \mathrm{~ms}$. For example, for this subject at all but the 2 highest intensities the PMR starts at a time before the $b$ wave reaches its peak amplitude.

The effect of PMR on ERGs elicited by a lowintensity $\left(-2 \cdot 16 \log \mathrm{cd} . \mathrm{s} . \mathrm{m}^{-2}\right)$ and a high-intensity $\left(-0.26 \log\right.$ cd. s. $\left.\mathrm{m}^{-2}\right)$ stimulus is shown in Figs. 10 , 11 , and 12. Fig. 10 shows a maximal-amplitude ERG (a) and an ERG elicited with about 2 log units less

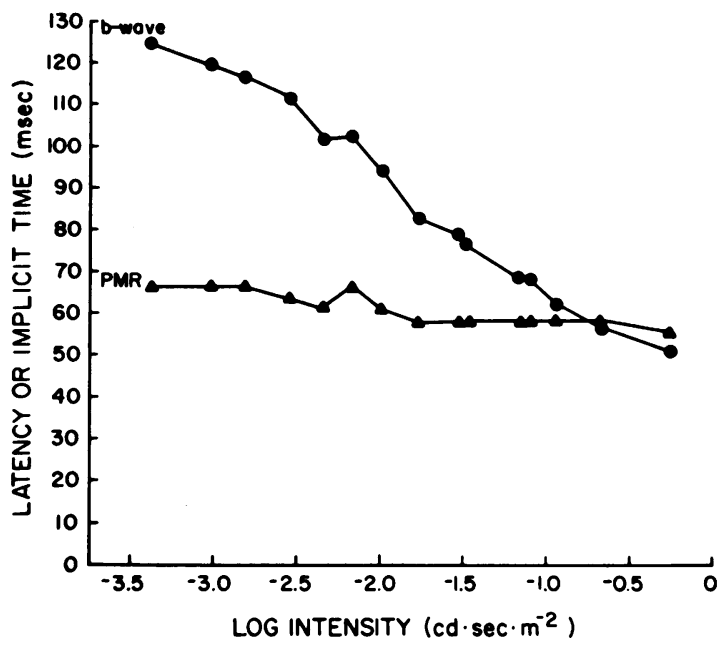

Fig. 9 Latency of the PMR and $b$ wave implicit time as a function of intensity in $\log \mathrm{cd}$. sec. $\mathrm{m}^{-2}$. Over a 3-log unit range of intensities the $b$ wave implicit time markedly decreased, while the PMR latency showed only a slight reduction. The graphs of the 2 functions cross at approximately $-0.7 \log c d . s . m^{-2}(57 \mathrm{~ms})$, indicating that for most of the dynamic range of the $b$ wave, the PMR will occur before the $b$ wave peak. 

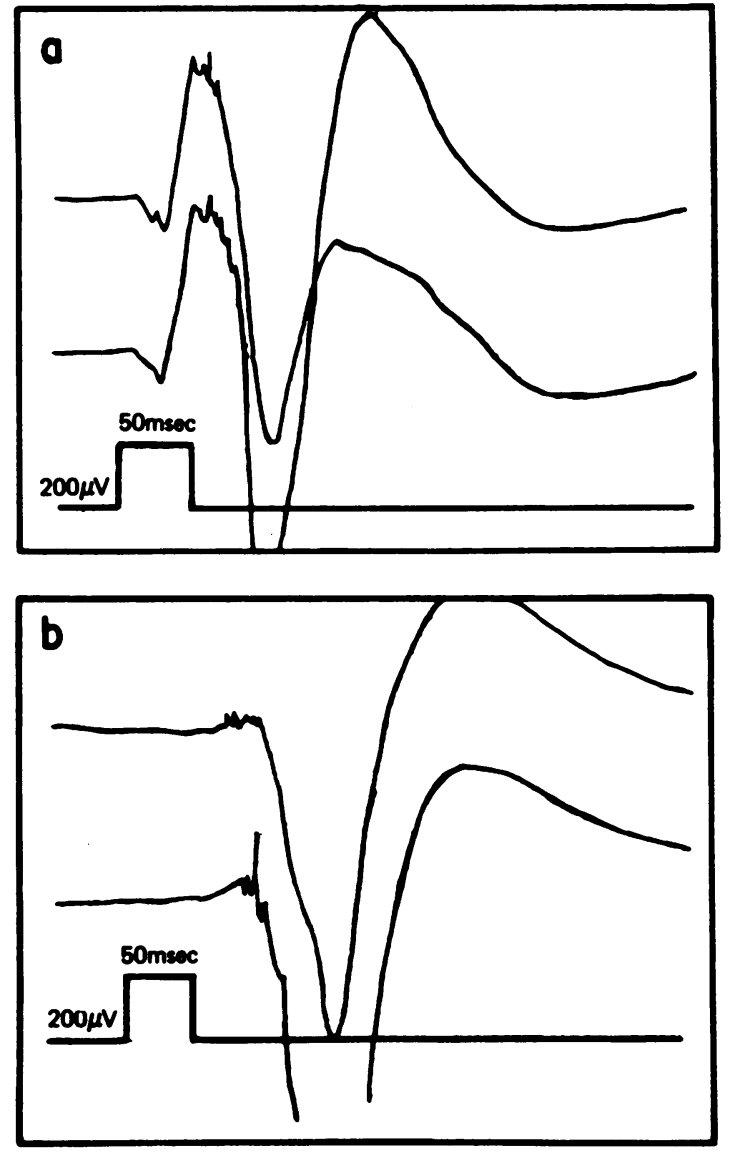

Fig. 10 The effect of the PMR on normal ERGs. (a) Two ERGs elicited by a stimulus intensity $\left(-0.26 \log \mathrm{cd} . \mathrm{s} . \mathrm{m}^{-2}\right)$ that produces a maximum amplitude ERG. (b) Two ERGs elicited by a stimulus intensity $\left(-2 \cdot 16 \log \mathrm{cd} . \mathrm{s} . \mathrm{m}^{-2}\right)$ that produces about a half-maximum amplitude ERG. The reflex completely obscures interpretation of these ERGs because of the different intensity-response characteristics of the ERG and PMR. The PMR would not become habituated in this subject.

intensity $(b)$ from a normal volunteer. In this case the reflex occurs slightly faster than for the subject reported above (Fig. 9), making measurement of the amplitude of the maximal-amplitude ERG difficult, and measurement of the amplitude of the loweramplitude ERG impossible (because the implicit time of the $b$ wave increases as the stimulus intensity decreases).

Fig. 11a illustrates ERGs from another subject in response to a light intensity that evoked a maximal amplitude ERG. Repeated, predictable deliveries of the stimulus habituated the reflex and eventually produced a totally time-locked $b$ wave response
(Figs. 11b and c). Fig. 12a illustrates 2 ERGs from the same subject, elicited by light of about 2 log units less intensity than that in Fig. 11. Note how the apparent implicit times of the $b$ waves changes with habituation of the PMR. Fig. 12b is a record of 10 consecutive responses to the same conditions after habituation of the reflex was achieved.

\section{POLARITY OF THE PMR}

We noticed in some cases that the polarity of the initial negative deflection of the PMR was reversed. The polarity of the pupillary response, however, remained corneal-positive in these individuals. PMRs of inverted polarity closely resembled ERGs of normal amplitude and delayed timing. The polarity change could be attributed to electrode position in relation to the standing field potential of the eye. If the initial position of the electrode was at the most positive pole of the eye, then any movements of the electrode or the eye would be expected to produce a negative deflection. In contrast, if the electrode's initial position was away from the most positive pole, then movements bringing the electrode position closer to the pole would be expected to produce a positive response. To test these possibilities the effect of initial ERG electrode position on the polarity of the response of voluntary eye movements was determined in the next experiment.

Procedure. The relative position of the electrode was changed by instructing the subject either to fixate a red LED directly in front of him, allowing the examiner to obtain central positioning of the contact lens on the cornea, or to fixate about $15^{\circ}$ above or below the LED, with his head still orientated directly forwards, allowing the examiner to obtain eccentric positioning of the contact lens in relation to the centre of the cornea. The subject was then instructed to execute a voluntary eye movement-either up, down, left, or right-when cued by a flash of light.

Results. A corneal-negative potential was recorded when the 2 subjects, fixating centrally, looked up. A similar potential was recorded when the subject directed his fixation from centre, either downwards or to the left or right. The potential was cornealpositive when the subject fixated eccentrically and moved his gaze to the centrally located LED. Thus, depending on the position of the contact lens in relation to the center of the cornea, normal eye blinks and movements of the eye under the electrode during ERG recording could result in either corneal-positive or corneal-negative PMRs.

Small corneal-positive PMRs, regardless of their origin, which occur in patients who have extinguished or severely reduced ERGs can easily be misinterpreted as being ERGs, because, as Karpe demonstrated, these PMRs can closely resemble retinal 
Fig. 11 The effect of habituation on the maximal amplitude ERG. (a) Two ERGs recorded from a wave form breaks down at the peak of the $b$ wave where the PMR begins. (b) Recorded during habituation, these 4 ERGs begin to show less variation in wave form. (c) The next 4 ERGs recorded are virtually superimposable because of the nearly total habituation of the PMR. normal subject. Note how the ERG
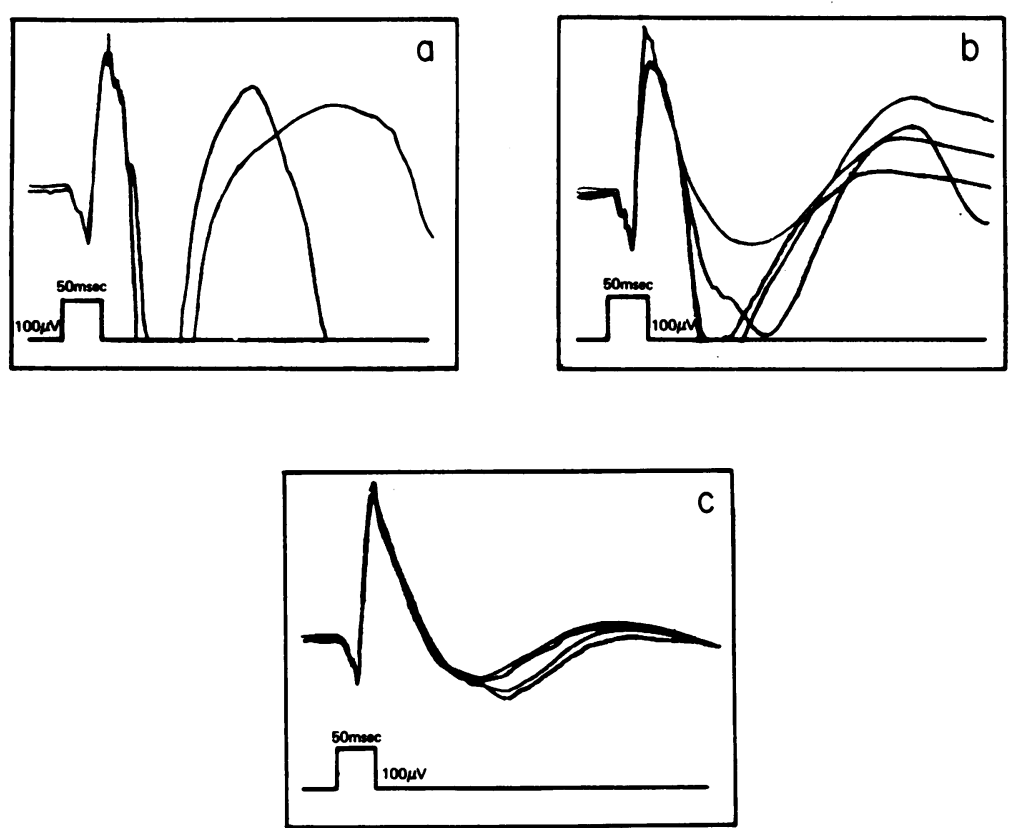

potentials. The epitome of this imitation is the clinical ERG shown in Fig. 13a, recorded from a patient with retinitis pigmentosa (RP). Because the response would not become habituated, it was not until the eye with the electrode was occluded and the fellow eye was stimulated (Fig. 13b) that the identity of the potential as a PMR was conclusively established. Note how closely this PMR resembles an ERG of normal amplitude but delayed timing.

Distortion of the ERG by the PMR has been observed similarly with various other patients. For example, the top trace of Fig. 14 shows the ERG

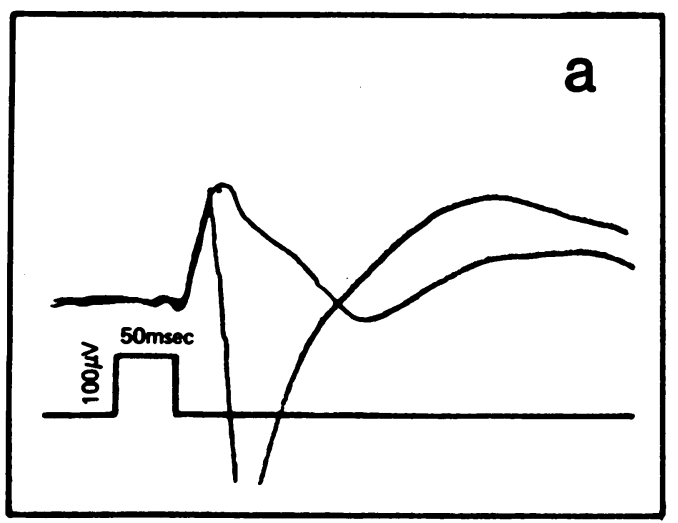

record obtained from another RP patient. The larger, slower potential could be interpreted as a delayed rod b wave, thus giving rise to abnormal splitting of rod and cone components. However, in this case habituation eliminated the PMR contribution to the potential, and the patient's actual ERG is shown in the middle trace of Fig. 14.

\section{EFFECT OF ELECTRODE TYPE ON THE} RECORDABILITY OF THE PMR

Several different types of electrodes are at present used to record the clinical ERG. We tested 3 of the

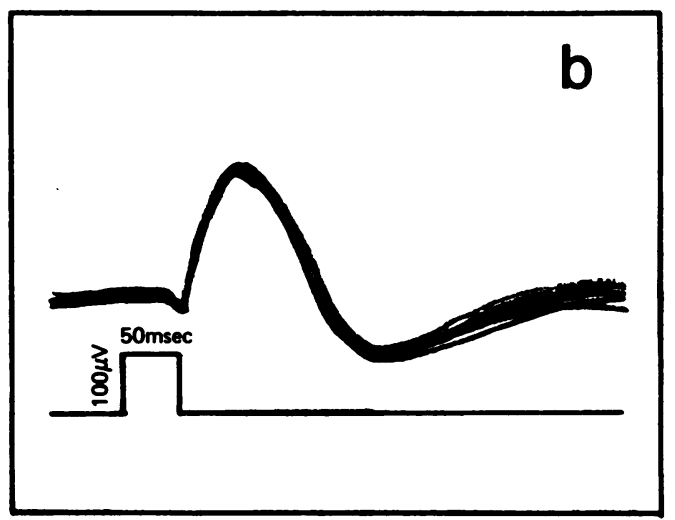

Fig. 12 The effect of habituation on a half-maximum amplitude ERG. (a) Two ERGs recorded from a normal subject using the lower-intensity $\left(-2 \cdot 16 \mathrm{og} . \mathrm{cd} . \mathrm{s} . \mathrm{m}^{-2}\right)$ stimulus. Note that the apparent implicit times of the $b$ wave change with habituation of the PMR. (b) Ten consecutive ERGs, recorded $3 \mathrm{~s}$ apart, after habituation of the PMR. The waveform is now totally time-locked to the stimulus. 

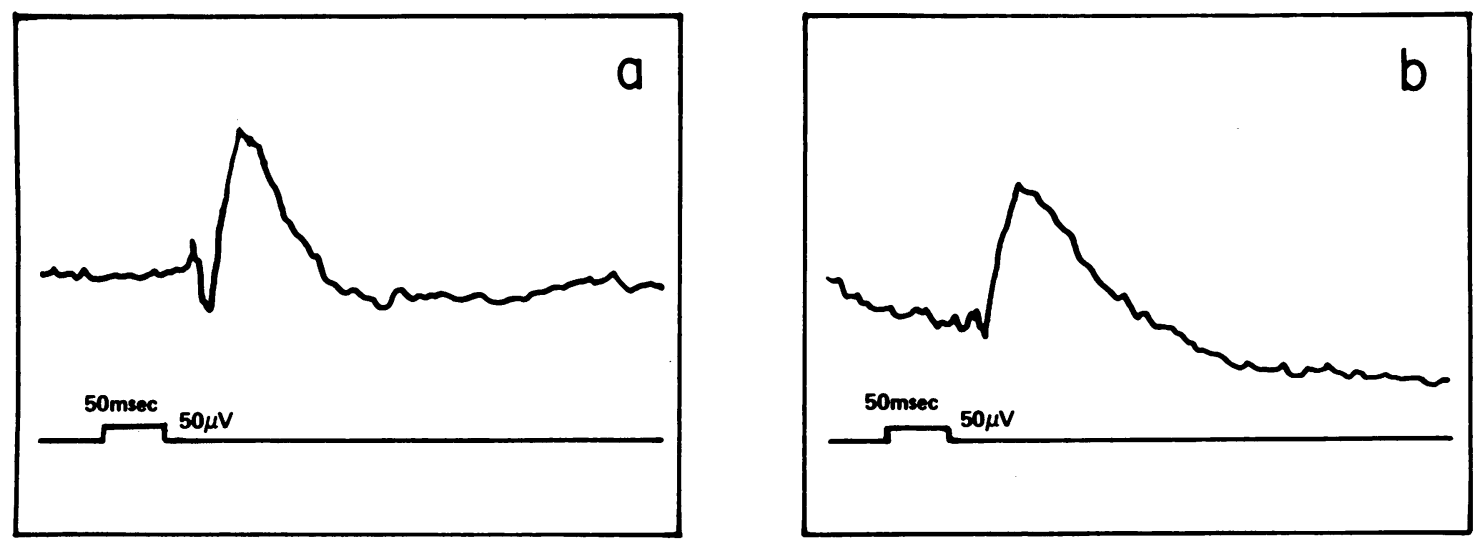

Fig. 13 The $P M R$ in retinitis pigmentosa $(R P)$. (a) This record was obtained during ERG testing of an $R P$ patient. The waveform resembles a normal amplitude ERG delayed in implicit time. (b) The eye containing the electrode was occluded, and the fellow eye was stimulated. Records obtained in this manner show that the waveform is artefactual.

most popular electrodes-the Burian-Allen bipolar electrode, the Burian-Allen monopolar electrode, and the gold foil electrode-comparing their sensitivity to recording the PMR.

Procedure. Because the Burian-Allen bipolar electrode was used throughout this study it became a reference in this experiment, recording the PMR in one eye while the gold foil or monopolar electrode recorded the PMR simultaneously in the occluded fellow eye. This manipulation permitted us to determine when the PMR was present and also to evaluate its recordability after habituation. The control experiments were performed, in which both eyes were occluded, to ensure that no light reached the experimental eye.

Results. The monopolar electrode recorded a lower amplitude PMR than was recorded by the bipolar electrode. This reduction in amplitude of the PMR appears to be mainly due to a reduction in recordability of the lid component; no extremely large PMRs were noted, and onset spiking was absent from most records. The main difference between the 2 electrodes is that the indifferent electrode is located in the speculum of the bipolar electrode, whereas it is placed on the patient's forehead for the monopolar electrode. Although the amplitude of the PMR

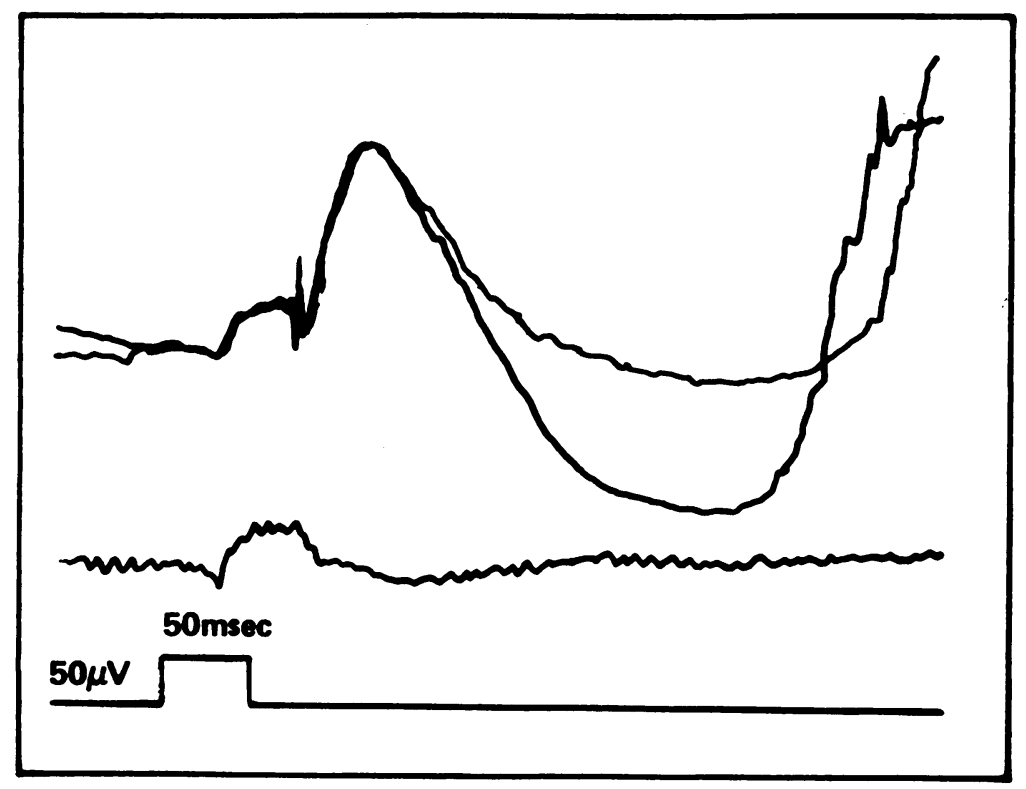

Fig. 14 The PMR in RP. The top trace shows 2 ERG traces obtained from an RP patient during standard $E R G$ testing. The larger, slower potential could be interpreted as a delayed rod $b$ wave, thus giving rise to abnormal splitting of rod and cone components. Habituation in this case eliminated the PMR and revealed the patient's actual ERG (middle trace). 
recorded by the monopolar electrode was reduced, the response often closely resembled a delayed ERG.

The gold foil electrode was very sensitive to recording the PMR, possibly because it touches the lower lid, and a strong medial movement of the lower lid occurs during actual or aborted blinks. ${ }^{10}$ The size of the PMR relative to that recorded with the bipolar electrode was quite variable during different sessions. This variability probably can be attributed to variable placement of the gold foil electrode and repositioning of the electrode by blinks.

Very little habituation was observed during testing of either the monopolar or gold foil electrode, despite habituation observed in the fellow eye with the bipolar electrode. In the case of the monopolar electrode lack of habituation may be due to the fact that the PMR recorded by this electrode was already quite small and may have mainly been due to the reflex eye movement. It is possible that the blink was becoming habituated, but the eye movement was not. In the case of the gold foil electrode even minor movements of the lower lid may have produced a sufficiently large response as to obscure signs of habituation.

In contrast it appears that the PMR cannot be recorded with the Henkes electrode. Van Norren (personal communication) indicated that he replicated the main experiments described here using the Henkes electrode, and when the experimental eye was occluded and the fellow eye stimulated no PMR could be recorded. Van Norren's failure to record the PMR probably can be attributed to the fact that the Henkes electrode is held on the cornea by a negative pressure; consequently the electrode moves with the eye and probably is not displaced by blinks.

\section{Discussion}

The present study has confirmed and described more fully an artefact that exists in the clinically recorded ERG. This artefact is a photomyoclonic reflex, consisting largely of eye lid movements and to a lesser extent eye movements. The mean latency of the PMR is approximately $59 \mathrm{~ms}$.

It is important to recognise the appearance of the PMR in ERG records, because it can interfere with the recording and interpretation of both the amplitude and the latency of the ERG. The eyelid movement reflex can be habituated by regular, repeated, predictable stimulus presentations in some but not all subjects, and habituation, when it occurs, may occur to a different extent among various subjects. The eye movement contribution to the reflex does not appear to become habituated as easily, and may thus subtly affect interpretation of low-amplitude or nonrecordable ERGs. Partially habituated lid movements may also insidiously interfere with ERG interpretation, especially if the polarity of the potential is corneal-positive.

Burian-Allen bipolar and gold foil electrodes are more susceptible to recording the artefact, the former because of the placement of the indifferent electrode in the speculum, the latter because it rests on the lower lid, which moves medially with every actual or attempted blink. ${ }^{10}$

Because recording the PMR may be unavoidable with most electrodes, it is important to recognise this spurious wave form in ERG records. Often, but not always, identification of the PMR when using a bipolar electrode is facilitated by observation of spiking activity at the onset of the reflex.

This work was supported by research and core facility grants EY01791 and EY -01765 from the National Eye Institute, and by a Research Center grant from the National Retinitis Pigmentosa Foundation.

We thank F. W. Fitzke, D. Guyton, A. Lasker, C. Perry, and D. Robinson for technical advice and assistance, D. Andrews and D. Finkelstein for comments on the manuscript, G. Fishman of the University of Illinois Eye and Ear Infirmary for the loan of the monopolar electrode, and D. van Norren of the Institute for Perception in Soesterberg, the Netherlands, for permitting us to cite his unpublished study.

\section{References}

1 Gur M, Zeevi Y. Frequency-domain analysis of the human electroretinogram. J Opt Soc Am 1980; 70: 53-9.

2 Bickford RG, White PL, Sem-Jacobsen CW, et al. Components of the photomyoclonic response in man. Fed Proc 1953; 12: 15 (abs).

3 Karpe G. The basis of clinical electroretinography. Acta Ophthalmol (Kbh) 1945; suppl 24: 1-128.

4 Miles WR. Eyeball reflex movement associated with voluntary and reflex winking. Am J Physiol 1925; 72: 239.

5 Pearlman JT. The c-wave of the human ERG: its intensity dependence and pupillociliary origin. Arch Ophthal 1962; 68: 823-30.

6 Marsh R, Hoffman HS, Stitt CL. Reflex inhibition audiometry. A new objective technique. Acta Otolaryngol (Stockh) 1978; 85: 336-41.

7 DelPezzo EM, Hoffman HS. Attentional factors in the inhibition of a reflex by a visual stimulus. Science $1980 ; 210$ : $673-4$.

8 Jayle GE, Boyer RL, Camo RL. L'Electrorétinographie Dynamique en Ophtalmologie. Paris: Masson, 1959.

9 Rushworth G. Observations on blink reflexes. J Neurol Neurosurg Psychiatry 1962; 25: 93-108.

10 Kennard DW, Glaser GH. An analysis of eyelid movements. J Nerv Ment Dis 1964; 139: 31-48.

11 Robinson DA. A method of measuring eye movement using a scleral search coil in a magnetic field. IEEE Trans Bio-Med Electronics 1963; 10: 137-45.

12 Collewijn H, van der Mark F, Jansen TC. Precise recording of human eye movements. Vision Res 1975; 15: 447-50. 